\title{
Comparison of Solid-State Dipolar Couplings and Solution Relaxation Data Provides Insight into Protein Backbone Dynamics
}

\author{
Veniamin Chevelkov, ${ }^{\dagger, \ddagger}$ Yi Xue, ${ }^{\ddagger}$ Rasmus Linser, ${ }^{\dagger}$ Nikolai R. Skrynnikov, ${ }^{*, \neq}$ and Bernd Reif ${ }^{*, \dagger}$ \\ Forschunginstitut für Molekulare Pharmakologie (FMP), Robert-Rössle-Strasse 10, 13125 Berlin, Germany, and \\ Department of Chemistry, Purdue University, 560 Oval Drive, West Lafayette, Indiana 47907-2084
}

Received November 19, 2009; Revised Manuscript Received January 24, 2010; E-mail: nikolai@ purdue.edu; reif@fmp-berlin.de

Internal motions on the scale of $10 \mathrm{~ns}-10 \mu \mathrm{s}$ fall in a "blind spot" of solution NMR experiments traditionally used for studies of protein dynamics. ${ }^{1}$ Recently, a new experimental strategy has emerged to probe these motions. In this new approach, the generalized order parameters derived from conventional ${ }^{15} \mathrm{~N}$ relaxation data are compared with their counterparts extracted from ${ }^{1} \mathrm{H}^{\mathrm{N}}-{ }^{15} \mathrm{~N}$ residual dipolar couplings. ${ }^{2,3}$ This method, however, is rather demanding in the part involving residual dipolar couplings (rDCs). The complete analysis of $\mathrm{rDC}$ requires a separation of structural and dynamic variables, as well as the variables pertaining to protein alignment in the orienting media. The success is dependent on the availability of accurate data from multiple aligned samples which need to be significantly different (orthogonal). Furthermore, the degree of alignment $A_{a}$ proves to be "entangled" with the amplitude of the (axially symmetric) internal motion, which makes it difficult to separate the two quantities. To address this situation, rDC-based order parameters have been scaled in relation to the relaxation-based order parameters. ${ }^{4}$ Recently, backbone rDCs such as ${ }^{13} \mathrm{C}^{\prime}-{ }^{13} \mathrm{C}^{\alpha}$ have been used to establish the scaling factor (corresponding dipolar vectors can be considered rigid or nearrigid for secondary-structure residues in small globular proteins). ${ }^{5,6}$

In this communication we propose an alternative approach where the solution-state rDC data are replaced with the solid-state DC data. Similar to rDC-based order parameters, DC-based order parameters absorb motions on a sub- $\mu$ s time scale. At the same time, the DC data have two critical advantages: (i) for protein molecules immobilized in the crystalline lattice the alignment tensor is known a priori ${ }^{7}$ and (ii) in a polycrystalline sample all vectors are rendered spatially equivalent as a consequence of powder averaging. Thus, the alignment variables and structural variables, which cause so much trouble in the analyses of dynamics using rDCs, do not enter into consideration in the case of the DC-based analyses.

The idea of comparing the DC-based order parameters, $S_{\mathrm{DC}}^{2}$, with the solution relaxation-based order parameters, $S_{\text {rel }}^{2}$, has been explored before. ${ }^{8}$ In this work, however, we benefit from the improved accuracy of the DC measurements. ${ }^{9}$ The advantage stems from the use of a perdeuterated protein sample with only $10 \%$ of protons back-substituted into exchangeable sites. For a small, wellbehaved protein such as $\alpha$-spectrin SH3 domain, ${ }^{10}$ this labeling scheme leads to high-quality spectra ${ }^{11}$ which, in turn, translate into more accurate DC data. It also helps that in the heavily deuterated sample one does not need to be concerned about proton-proton couplings. ${ }^{12}$ The CPPI recoupling sequence ${ }^{13}$ employed in our experiment is remarkably stable with respect to $\mathrm{rf}$ field mismatch and inhomogeneity, which further contributes to the accuracy of the measurements. Finally, certain progress has been made on the

\footnotetext{
Forschunginstitut für Molekulare Pharmakologie.

* Purdue University.
}

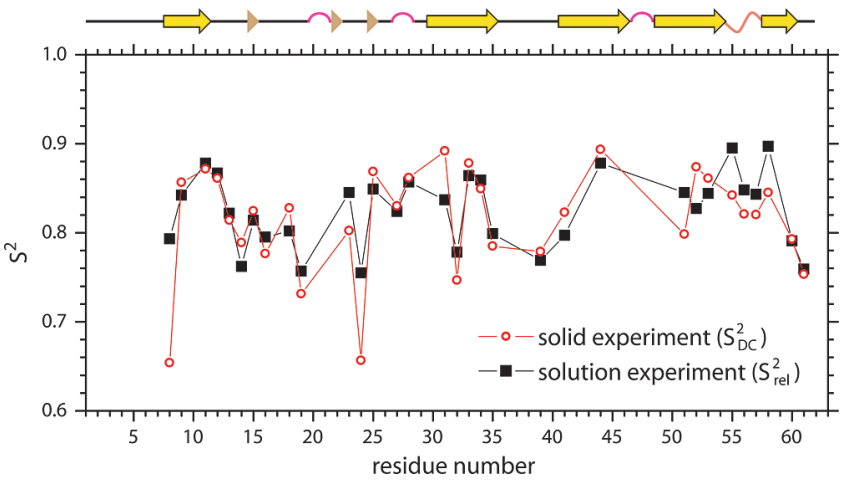

Figure 1. Experimentally determined order parameters in $\alpha$-spectrin SH3. The solid- and solution-state data sets have been analyzed independently using the standard values for the $\mathrm{NH}$ bond length, $r_{\mathrm{NH}}=1.02 \AA$, and the ${ }^{15} \mathrm{~N}$ CSA anisotropy, $-172 \mathrm{ppm}$. Using the more accurate estimate for the effective bond length, $1.015 \AA$, ${ }^{5}$ leads to a trivial downward shift in the $S_{\mathrm{DC}}^{2}$ and $S_{\text {rel }}^{2}$ profiles ( $1.5 \%$ and $\sim 2.3 \%$, respectively). Only those residues for which both solid and solution data are available are shown in the plot (see Table S1 for complete list of data).

interpretation side; e.g., the proton isotropic chemical shift has been explicitly included in the simulations. ${ }^{9}$ As a result of all these improvements, the estimated average error in the DCs has been cut to the level of ca. $3 \% .{ }^{14}$ This compares favorably with the previous body of work where the errors were in excess of $5 \%$ and usually close to $10 \%$. This kind of improvement is critical for the analysis of the DC-derived order parameters, which are normally confined to a relatively narrow range.

The solid-state DC data described above were converted into order parameters, $S_{\mathrm{DC}}^{2}$, using the simple relationship $D_{i}=S_{\mathrm{DC}} D_{\max }$, where $D_{\max }=-(1 / 2 \pi)\left(\mu_{0} / 4 \pi\right) \gamma_{\mathrm{H}} \gamma_{\mathrm{N}} \hbar / r_{\mathrm{NH}}^{3}$. Note that this approach ignores the anisotropy of local motion, which does not manifest itself in our experimental data ${ }^{9}$ (see Supporting Information for detailed discussion). Separately and independently, ${ }^{15} \mathrm{~N}$ relaxation data from our previous solution-state study $\left(R_{1}, R_{2}\right.$, NOE measured at 500 and $600 \mathrm{MHz}$ field strengths) ${ }^{15}$ were analyzed by means of the standard two-parameter Lipari-Szabo model. ${ }^{16}$ The resulting fast-motion parameters are typical of a small globular protein such as $\alpha$-spectrin SH3 (mean $S_{\text {rel }}^{2}=0.83, \tau_{\mathrm{e}}=27 \mathrm{ps}$ ).

Figure 1 illustrates the comparison between the solid-state $S_{\mathrm{DC}}^{2}$ and solution $S_{\text {rel }}^{2}$. The agreement between the two independently obtained series of order parameters is remarkable. Aside from the two residues, Leu- 8 and Thr-24, $S_{\mathrm{DC}}^{2}$ and $S_{\text {rel }}^{2}$ values are nearly identical, strongly suggesting that most amide sites do not experience any significant motions on the ns $-\mu$ s time scale. ${ }^{17}$

This picture is consistent with the consensus that has recently emerged in the field. Specifically, the rDC data from select globular proteins (ubiquitin and GB3) suggest that there is very little or no $\mathrm{ns}-\mu \mathrm{s}$ motions in the protein backbone-aside from termini and a 
handful of mobile loops. ${ }^{3,6}$ This notion is also in agreement with early relaxation studies that focused on ns dynamics. ${ }^{18}$

Of special interest for us is comparing the results in Figure 1 with our previous findings based on the combination of solutionand solid-state relaxation data. In particular, we recently analyzed a large set of ${ }^{15} \mathrm{~N}$ data from the $\alpha$-spectrin $\mathrm{SH} 3$ domain, which included the solid-state $R_{1}$ rates measured at 600 and $900 \mathrm{MHz}{ }^{15}$ The analysis, using a variant of the extended Lipari-Szabo model, allowed us to probe the motions on the time scale $\sim 1-100 \mathrm{~ns}$. As it turned out, for 19 out of 34 characterized residues the slowmotion order parameter $S_{\mathrm{rel}, \mathrm{s}}^{2}$ is greater than 0.97 . Furthermore, for all but two residues $S_{\text {rel,s }}^{2}$ is greater than $0.92 .{ }^{19}$ This result points toward the small amplitudes of ns motions, in agreement with the data shown in Figure 1. Considering residues Leu- 8 and Thr-24 which show a significant difference between DC- and relaxationbased order parameters, Figure 1, both of them produced evidence of ns motion in our previous study ( $S_{\text {rel,s }}^{2}$ equal to 0.92 and 0.93 , respectively). ${ }^{15}$ Similar observations can be made by analyzing the extended set of the solid-state data. ${ }^{20}$

In the absence of the overall tumbling, ns $-\mu$ s local dynamics in solids can be highly efficient in causing spin relaxation, which in turn leads to line broadening and the ultimate disappearance of spectral peaks. In $\alpha$-spectrin SH3, this happens to residues $1-7$ from the disordered $\mathrm{N}$-terminus, residues $36-38$ from the so-called n-Src loop, and residues 47-48 from the distal loop. The corresponding spectral peaks are weak or missing in the current DC experiment which employs cross-polarization transfer (although some of them show up in the INEPT-based experiment; see Figure $\mathrm{S} 1)$. Due to dynamic disorder, the $\mathrm{N}$-terminal residues and several atoms in Asp-48 also cannot be resolved by X-ray crystallography. ${ }^{21}$ Note that these observations fit the general pattern whereby ns $-\mu \mathrm{s}$ dynamics is concentrated in the loop regions and termini.

Dipolar relaxation effectively restricts the range of dynamics that can be probed through solid-state DCs (upper limit of several microseconds, Figure S2). In contrast, rDCs in solution impose no such restriction because they are typically 1000 -fold smaller than DCs. In practice, rDCs can be used to study motions on the time scale extending to several milliseconds. There is, therefore, a potentially useful complementarity between the two types of data, with DCs being more selective of the two.

A meaningful comparison between $S_{\text {rel }}^{2}$ and $S_{\mathrm{DC}}^{2}$ is predicated on the assumption that backbone dynamics does not change significantly upon protein crystallization. ${ }^{15}$ To further explore this aspect, we undertook a series of MD simulations. Briefly, the crystallographic unit cell containing four protein molecules has been constructed (protein coordinates are from high-resolution structure 1U06; the dimensions of the orthorhombic unit cell are from roomtemperature structure 2NUZ). ${ }^{21}$ The cell was then hydrated and used as the initial structural model to record a 50-ns MD trajectory (CHARMM 32b2). ${ }^{22}$ The simulation protocol was the same as previously described for the trajectory of $\alpha$-spectrin $\mathrm{SH} 3$ in solution. ${ }^{15,23}$ The only difference arises from the definition of the water box and the associated periodic boundary conditions: in one case the model recreates the crystal lattice environment, including multiple crystal contacts, while in the other case the protein is completely immersed in water.

The solid-state order parameters have been extracted from the MD trajectory as ${ }^{24}$

$$
S^{2}=(4 \pi / 5) \sum_{m=-2}^{2}\left\langle Y_{2 m}^{*}(\Omega)\right\rangle\left\langle Y_{2 m}(\Omega)\right\rangle
$$

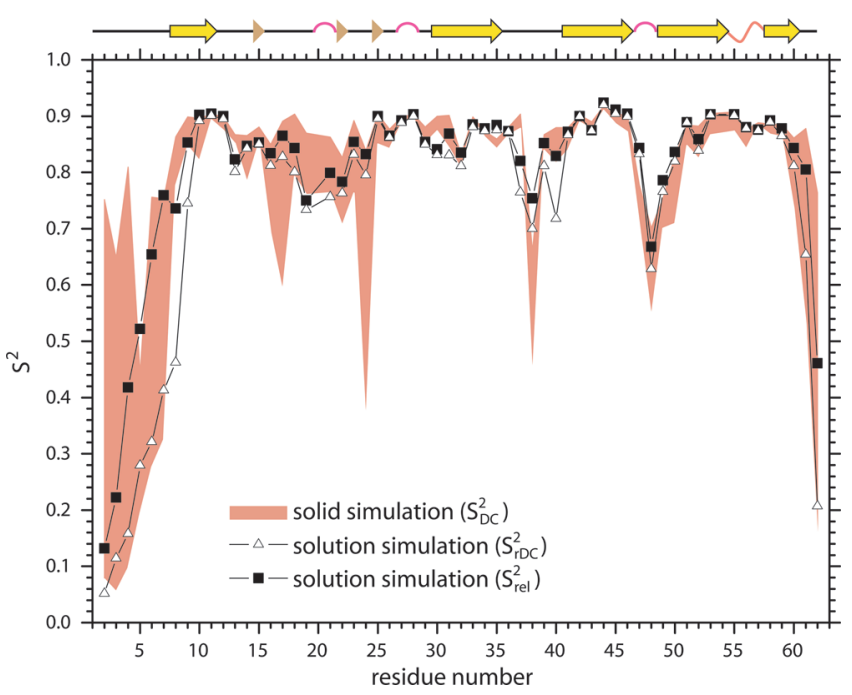

Figure 2. Simulated order parameters in $\alpha$-spectrin SH3. Of note, the correlation coefficient between the simulated and experimental $S_{\mathrm{DC}}^{2}$ values turns out to be $r=0.94$ (calculated for 44 residues for which the experimental solid-state data are available; simulated $S_{\mathrm{DC}}^{2}$ values have been averaged over four protein molecules in the unit cell).

where $\Omega$ describes the direction of the NH vector in the fixed crystal frame. So-defined order parameters absorb both ps and ns dynamics and therefore can be thought of as the analogue of $S_{\mathrm{DC}}^{2}$. The range of the simulated $S_{\mathrm{DC}}^{2}$ values from the four protein molecules in the unit cell is represented with a pink shaded area in Figure 2. Equation 1 has also been applied to the solution MD data, post-processed to remove the effect of the protein's overall tumbling (simulated order parameters $S_{\mathrm{rDC}}^{2}$; open triangles in Figure 2). Alternatively, the solution trajectory can be used to simulate the set of ${ }^{15} \mathrm{~N}$ relaxation rates (comprised of the same data as the experimental data set), that are subsequently fitted with the Lipari-Szabo model. The resulting order parameters are equivalent to $S_{\text {rel }}^{2}$ (black squares in Figure 2).

Even though the current MD trajectories are relatively short, they offer a number of interesting insights. Through most of the sequence the solution data show little or no ns dynamics (triangles vs squares), with the pattern of the order parameters similar to that observed in the hydrated crystal (shaded area). In addition, a number of observations pertaining to the specific sites can be made.

(i) In solution, the disordered $\mathrm{N}$-terminus (residues $1-7$ ) engages in large-amplitude motions on the ns time scale. In solids, these motions are hindered/slowed down by extensive crystal contacts. Consequently, large-scale transitions have been observed for some, but not all, of the four protein molecules comprising the crystallographic unit cell (resulting in a wide spread of $S_{\mathrm{DC}}^{2}$ values, Figure 2).

(ii) Residue Leu- 8 , which caps the $\beta$-sheet, is also affected. The solution trajectory shows that occasionally the hydrogen bond at this site becomes broken and Leu- 8 starts behaving as a part of the disordered N-terminus. This explains the presence of ns $-\mu \mathrm{s}$ dynamics in this residue (Figure 1). In the crystalline environment, however, large fluctuations involving Leu-8 apparently become too infrequent and therefore do not register in the relatively short solidstate trajectory. In the case of the other dynamic residue, Thr-24, both solution and solid-state simulations reproduce the respective experimental parameters, $S_{\text {rel }}^{2}$ and $S_{\mathrm{DC}}^{2}$, reasonably well.

(iii) Curiously, the mobility of residue Asn-38 increases upon crystallization. As it turns out, the corresponding peptide plane jumps between two disparate orientations. One of the two conformations is somewhat strained $\left(\psi \approx 20^{\circ}\right.$ in Thr-37) and is not 
favored in solution. In the crystal, however, this conformation is stabilized by the intermolecular hydrogen bonds formed by the side chain of Asn-38. This is an interesting example of how crystal contacts can lead to a local destabilization in the protein structure. Recall that residue 38 is unobservable in the solid-state DC experiment.

(iv) The presence of significant dynamics in the distal loop around Asp-48 is confirmed by the increased crystallographic B-factors. Residue Asn-47 assumes an unusual conformation $\left(\phi \approx 50^{\circ}, \psi \approx\right.$ $\left.-110^{\circ}\right)^{25}$ which has a destabilizing effect on the structure. ${ }^{26}$ Asp48 is also the only residue featuring exchange broadening in solution $\left(R_{\mathrm{ex}}=28 \mathrm{~s}^{-1}\right.$ at $\left.600 \mathrm{MHz}\right)$.

It can be safely assumed that ns $-\mu$ s dynamics in a protein backbone involves crossing energy barrier(s). For example, conformational rearrangements in short peptides involving transitions over multiple barriers in $(\phi, \psi)$-space occur on this time scale. ${ }^{27}$ In this sense $n s-\mu$ s dynamics is similar to the extensively studied $\mu \mathrm{s}-\mathrm{ms}$ dynamics. As it happens, $\mu \mathrm{s}-\mathrm{ms}$ motions are rare in wellstructured globular proteins. When such motions occur, they mostly involve loops, ${ }^{28}$ as well as special situations like local unfolding, ${ }^{29}$ disulfide or proline isomerization, ${ }^{30}$ etc. We suggest that the same is true for ns $-\mu$ s backbone motions - they are mostly confined to the loop regions and are unlikely to occur in the structured portion of a protein, e.g. in a $\beta$-pleated sheet.

Indeed, it is difficult to expect that a globular protein would alternate between two similar but distinct conformational states with appreciably different $\beta$-sheet geometries (i.e., significantly different orientations of peptide planes within the $\beta$-sheet). Such a scenario would be inconsistent with Anfinsen's dogma which suggests that the protein fold is unique. ${ }^{31}$ While it is known that side chains are frequently engaged in rotameric jumps on the time scale $>1 \mathrm{~ns}$, recent studies show that these motions do not affect the structured portion of the backbone which "nearly always...stays within a single local energy well", 32

In conclusion, our results suggest that comparative analysis of solution relaxation data and solid-state DCs can provide a surprisingly accurate picture of backbone dynamics. In particular, this approach can be used to access much-discussed ns $-\mu$ s backbone motions. In this respect, solid-state DCs offer a new route compared to solution rDCs. Our data support the emerging point of view that ns $-\mu$ s motions occur in mobile loops, termini, and side chains, but generally not in the structured portion of the backbone. Scaffolds such as the $\beta$-sandwich are apparently too stiff, exhibiting fast (ps) motions, but not conducive to slower dynamics. It is clear, however, that more proteins with different architectures need to be studied before any broad conclusions can be drawn.

Acknowledgment. This work was supported by the DFG grant Re1435 to B.R. and the NSF grant CHE-0723718 to N.R.S. We are grateful to Nathan Benjamin for his help with MD simulations.

Supporting Information Available: Solid-state spectra of $\alpha$-spectrin SH3 domain; plot illustrating the range of $\left\{S^{2}, \tau\right\}$ amenable to DC-based studies; equivalent of Figure 1 with indicated experimental uncertainties; complete table of $S_{\mathrm{DC}}^{2}$ and $S_{\text {rel }}^{2}$ data; analysis of the effect of motional anisotropy on $S_{\mathrm{DC}}^{2}$; description of the MD simulation protocol. This material is available free of charge via the Internet at http://pubs.acs.org.

\section{References}

(1) Palmer, A. G. Chem. Rev. 2004, 104, 3623.

(2) Tolman, J. R.; Al-Hashimi, H. M.; Kay, L. E.; Prestegard, J. H. J. Am. Chem. Soc. 2001, 123, 1416. Meiler, J.; Prompers, J. J.; Peti, W.; Griesinger, C.; Brüschweiler, R. J. Am. Chem. Soc. 2001, 123, 6098. Clore, G. M.; Schwieters, C. D. Biochemistry 2004, 43, 10678.

(3) Yao, L.; Vögeli, B.; Torchia, D. A.; Bax, A. J. Phys. Chem. B 2008, 112, 6045. Lange, O. F.; Lakomek, N. A.; Farès, C.; Schröder, G. F.; Walter, K. F. A.; Becker, S.; Meiler, J.; Grubmüller, H.; Griesinger, C.; de Groot, B. L. Science 2008, 320, 1471.

(4) Tolman, J. R. J. Am. Chem. Soc. 2002, 124, 12020. Lakomek, N. A.; Walter, K. F. A.; Farès, C.; Lange, O. F.; de Groot, B. L.; Grubmüller, H.; Brüschweiler, R.; Munk, A.; Becker, S.; Meiler, J.; Griesinger, C. J. Biomol. NMR 2008, 41, 139.

(5) Yao, L. S.; Vögeli, B.; Ying, J. F.; Bax, A. J. Am. Chem. Soc. 2008, 130, 16518.

(6) Salmon, L.; Bouvignies, G.; Markwick, P.; Lakomek, N.; Showalter, S.; Li, D. W.; Walter, K.; Griesinger, C.; Bruschweiler, R.; Blackledge, M. Angew. Chem. Int. Ed. 2009, 48, 4154.

(7) In principle, one should take into consideration a possibility of "rocking" motion of the protein in the crystal cell. There is, however, evidence that the amplitude of this motion is very small, on the order of $1^{\circ}$ (Meinhold, L.; Smith, J. C. Biophys. J. 2005, 88, 2554). At this point we choose to disregard this motion.

(8) Cole, H. B. R.; Torchia, D. A. Chem. Phys. 1991, 158, 271. Lorieau, J. L.; McDermott, A. E. J. Am. Chem. Soc. 2006, 128, 11505. Yang, J.; Tasayco, M. L.; Polenova, T. J. Am. Chem. Soc. 2009, 131, 13690.

(9) Chevelkov, V.; Fink, U.; Reif, B. J. Am. Chem. Soc. 2009, 131, 14018.

(10) Castellani, F.; van Rossum, B.; Diehl, A.; Schubert, M.; Rehbein, K.; Oschkinat, H. Nature 2002, 420, 98.

(11) Chevelkov, V.; Rehbein, K.; Diehl, A.; Reif, B. Angew. Chem., Int. Ed. 2006, 45,3878

(12) Munowitz, M.; Aue, W. P.; Griffin, R. G. J. Chem. Phys. 1982, 77, 1686.

(13) Dvinskikh, S. V.; Zimmermann, H.; Maliniak, A.; Sandstrom, D. J. Magn. Reson. 2003, 164, 165. Dvinskikh, S. V.; Zimmermann, H.; Maliniak, A.; Sandstrom, D. J. Chem. Phys. 2005, 122, 044512.

(14) Includes random noise-type error (see Figure S1), as well as ca. $1 \%$ systematic error which originates mainly from site-to-site variations in the ${ }^{15}$ N CSA anisotropy?

(15) Chevelkov, V.; Zhuravleva, A. V.; Xue, Y.; Reif, B.; Skrynnikov, N. R. J. Am. Chem. Soc. 2007, 129, 12594.

(16) Lipari, G.; Szabo, A. J. Am. Chem. Soc. 1982, 104, 4546.

(17) It is assumed that internal protein dynamics in a well-hydrated protein crystal is quantitatively similar to dynamics in solution, notwithstanding the effect of crystal contacts (Agarwal, V.; Xue, Y.; Reif, B.; Skrynnikov, N. R. J. Am. Chem. Soc. 2008, 130, 16611). An equivalent assumption is made in the rDC-based studies, where interactions between protein and alignment media can potentially alter a protein's native dynamics (Louhivuori, M.; Otten, R.; Lindorff-Larsen, K.; Annila, A. J. Am. Chem. Soc. 2006, 128, 4371).

(18) Clore, G. M.; Driscoll, P. C.; Wingfield, P. T.; Gronenborn, A. M. Biochemistry 1990, 29, 7387.

(19) This statistics is slightly different from the one reported in ref 15 due to several corrected spectral assignments. The conclusions of the article remain unaffected.

(20) Chevelkov, V.; Fink, U.; Reif, B. J. Biomol. NMR 2009, 45, 197.

(21) Chevelkov, V.; Faelber, K.; Diehl, A.; Heinemann, U.; Oschkinat, H.; Reif, B. J. Biomol. NMR 2005, 31, 295

(22) Brooks, B. R.; et al. J. Comput. Chem. 2009, 30, 1545.

(23) Xue, Y.; Pavlova, M. S.; Ryabov, Y. E.; Reif, B.; Skrynnikov, N. R. J. Am. Chem. Soc. 2007, 129, 6827.

(24) Brüschweiler, R.; Wright, P. E. J. Am. Chem. Soc. 1994, 116, 8426.

(25) Vega, M. C.; Martínez, J. C.; Serrano, L. Protein Sci. 2000, 9, 2322.

(26) Martinez, J. C.; Pisabarro, M. T.; Serrano, L. Nat. Struct. Biol. 1998, 5, 721.

(27) Krieger, F.; Fierz, B.; Bieri, O.; Drewello, M.; Kiefhaber, T. J. Mol. Biol. $\mathbf{2 0 0 3}, 332,265$

(28) Ishima, R.; Freedberg, D. I.; Wang, Y. X.; Louis, J. M.; Torchia, D. A. Structure 1999, 7, 1047. Rozovsky, S.; McDermott, A. E. J. Mol. Biol. 2001, 310, 259 .

(29) Mulder, F. A. A.; Mittermaier, A.; Hon, B.; Dahlquist, F. W.; Kay, L. E. Nat. Struct. Biol. 2001, 8, 932.

(30) Loria, J. P.; Rance, M.; Palmer, A. G. J. Am. Chem. Soc. 1999, 121, 2331. Eisenmesser, E. Z.; Millet, O.; Labeikovsky, W.; Korzhnev, D. M.; WolfWatz, M.; Bosco, D. A.; Skalicky, J. J.; Kay, L. E.; Kern, D. Nature 2005, $438,117$.

(31) Anfinsen, C. B. Science 1973, 181, 223.

(32) Davis, I. W.; Arendall, W. B.; Richardson, D. C.; Richardson, J. S. Structure 2006, 14, 265 .

JA100645K 